\title{
Shave-Exzisionen dysplastischer Nävuszellnävi: Methodisches Vorgehen
}

\author{
Shave Excisions of Dysplastic Nevi: Methodical Approach
}

Autor

Institut

\section{Deichmann}

Hautarztpraxis Gelnhausen und Universitäts-Hautklinik Heidelberg

\section{Bibliografie}

DOI $10.1055 / \mathrm{s}-2008-1077380$

Akt Dermatol 2008; 34:

279-281 @ Georg Thieme

Verlag KG Stuttgart · New York ISSN 0340-2541

Korrespondenzadresse

Prof. Dr. med.

Martin Deichmann

Hautarztpraxis

Am Ziegelturm 4

63571 Gelnhausen

hautarzt@deichmann.info

\section{Zusammenfassung \\ $\nabla$}

Die Exzision des dysplastischen Nävuszellnävus als häufigste Operation des Dermatologen kann minimal-invasiv als Shave-Exzision durchgeführt werden. Im vorliegenden Beitrag werden technische Aspekte besprochen: So wird die Dermis unmittelbar zuvor mit Lokalanästhetikum aufgepolstert, um die richtige Schnittebene zu treffen, nicht zu flach wegen der Rezidivgefahr, nicht zu tief wegen Narbenbildung. Eine rasche Stillung der Punktblutungen ist mit AluminiumchloridLösung (Mallebrin ${ }^{\circledR}$ ), die Wundbehandlung mit

\section{Einleitung \\ $\nabla$}

Minimal-invasive Verfahren werden von Patienten vermehrt nachgefragt, so z. B. die photodynamische Therapie von Basaliomen statt der Exzision oder die Schaum-Sklerosierung unter duplexsonographischer Kontrolle bei Stammvarikosis statt Crossektomie mit Stripping. Auch bei der häufigsten Operation des Dermatologen, der Exzision dysplastischer Nävuszellnävi (NZN) zeigt sich der Trend zu einem minimal invasiven Operations-Verfahren: Shave-Exzisionen finden auch in Deutschland zunehmend Anwendung, weil sie unmittelbar nach einer Hautkrebs-Vorsorgeuntersuchung schnell und einfach durchgeführt werden können, vorausgesetzt die Haftpflichtversicherung des Arztes verlangt keine 24 Stunden Bedenkzeit vor einem operativen Eingriff. Dass in der dermatologischen Literatur technische Aspekte dieses gängigen Operationsverfahrens bisher wenig diskutiert wurden [2], erstaunt daher. Hierzu will der Autor beitragen, indem das eigene Vorgehen beschrieben und zur Diskussion gestellt wird.
Fusidinsäure-Salbe (Fucidine ${ }^{\circledR}$ ) möglich. Marcumar ${ }^{\circledR}$-Pause mit Heparinisierung, Vollnarkose bei Kleinkindern oder postoperatives Sport-Verbot sind bei Shave-Exzisionen entbehrlich. Wird histologisch Dysplasie beschrieben mit zusätzlicher Randbildung, wird die OP-Stelle in $2-3$ Monaten kontrolliert und der Patient über die Rezidiv-Gefahr aufgeklärt. Das weniger traumatisierende Verfahren der Shave-Exzision ist unmittelbar im Anschluss einer Hautkrebs-Vorsorgeuntersuchung mit geringem Aufwand möglich und führt in der Hand des geschickten Dermatologen zumeist zu gutem ästhetischen Ergebnis.

\section{Vereinfachtes „OP-Päckchen“ $\nabla$}

Da bei der Shave-Exzision die Haut nur bis zum Korium traumatisiert wird, ähnlich einer Schürfwunde, genügen verminderte Anforderungen an die Sterilität. Statt eines großen sterilen OP-Päckchens genügt ein sauberes Stoff- oder Papiertuch von der Rolle, darauf eine sterile Kompresse, darauf eine sterile Skalpellklinge mit abgerundeter Schneidefläche. Statt steriler OP-Handschuhe ist es ausreichend, Einmal-Handschuhe anzuziehen mit nachfolgender Desinfektion. Wie bei konventioneller Exzision unterschreibt der Patient eine Aufklärung, in der über die üblichen Komplikations-Risiken wie Wundinfektion und Narbenbildung aufgeklärt wird.

\section{„Aufpolsterung“ durch Lokal- anästhesie \\ $\nabla$}

Entscheidend bei der Shave-Exzision ist die richtige Schnitt-Ebene, um den NZN vollständig zu exzidieren, bei Reepithelialisierung möglichst ohne Narbenbildung. Relativ unproblematisch ist dies am Rücken mit kräftigem Korium möglich, weshalb sich insbesondere der Rücken zum 


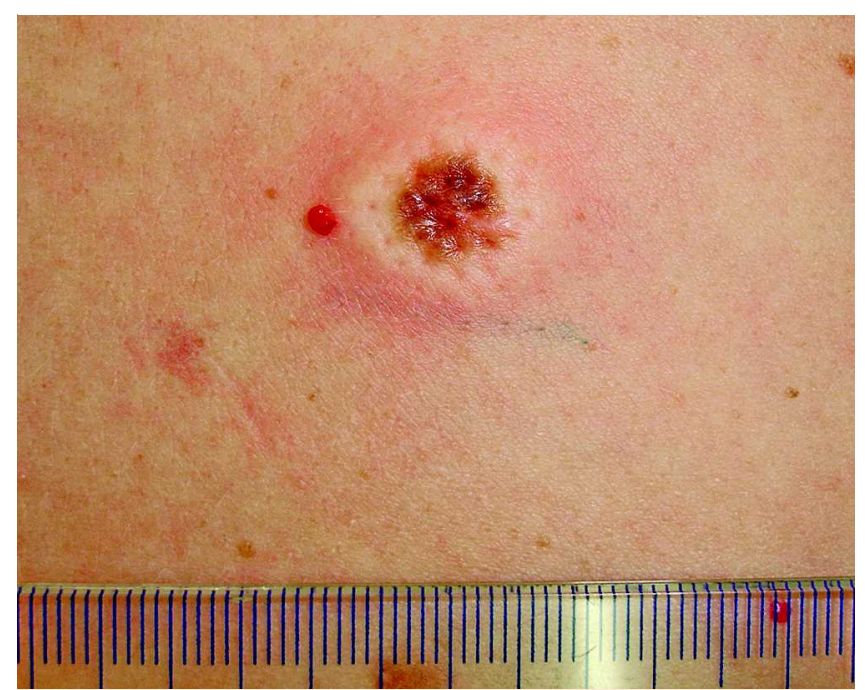

Abb. 1 „Aufpolsterung“ der Dermis durch die Lokalanästhesie.

Einüben der Operationstechnik eignet. Die richtige Schnittebene wird leichter getroffen durch unmittelbar vorherige oberflächliche „Aufpolsterung“ der Dermis mit Lokalanästhetikum ( $\bullet$ Abb.1). Eine 2-ml-Spritze eignet sich hierzu besser wegen des erhöhten Stempeldruckes. Ein Adrenalin-Zusatz in der Lokalanästhesie ist entbehrlich. Lokalisationen mit dünnem Korium wie z.B. Mammae oder Sprunggelenke eignen sich für die Shave-Technik eher weniger.

\section{Schnittfïhrung}

\section{$\nabla$}

Wenn die Haut durch die oberflächliche Lokalanästhesie hart und weißlich ist, wird unmittelbar mit der Exzision begonnen. Mit der einen Hand wird eine Hautfalte gehalten, mit der anderen Hand die Skalpellklinge wie eine Säge hin- und herbewegt: Im Abstand von etwa 1-2 Millimeter vom NZN entfernt erfolgt die Inzision der gesunden Haut initial mit einem größeren Schnittwinkel von ca. 50-70\%. Der Schnitt wird zunächst im Uhrzeigersinn erweitert ( $\bullet$ Abb. 2), danach gegen den Uhrzeigersinn. Hiernach kann die Läsion auf ganzer Breite mit flachem Schnittwinkel exzidiert werden. Die richtige Schnittebene zu treffen, nicht zu flach wegen Rezidivgefahr, nicht zu tief wegen Narbenbildung, ist die eigentliche Kunst der Shave-Exzision.

\section{Abschätzung des Rezidiv-Risikos $\nabla$}

Das Exzidat von der Schnittfläche betrachtet sollte durchgängig weißlich sein, dann ist eine vollständige Exzision des NZN wahrscheinlich. Zeigt die weißliche Exzidat-Unterseite eine bräunliche oder schwärzliche Unterbrechung, ist im histologischen Befund die Beschreibung einer Randbildung und klinisch ein NZN-Rezidiv zu erwarten. In diesem Fall kann der Patient in drei Monaten zu einer Kontrolle der Shave-Stelle einbestellt werden. Ein eventuelles Rezidiv tritt meistens im Zentrum der Shave-Stelle auf und kann nochmals durch Shave-Exzision entfernt werden. Kommt es ausnahmsweise zum nochmaligen Rezidiv, erfolgt die tiefe Exzision mit Subkutan- und EinzelknopfNähten, die dann aber meist kleiner ausfällt als bei initialer Spindelexzision.

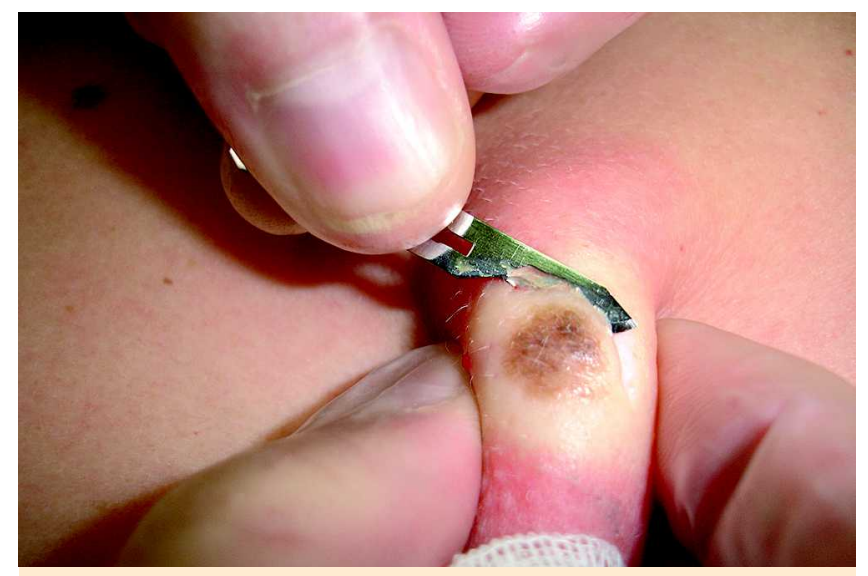

Abb. 2 Schnittführung mit initial größerem Schnittwinkel.

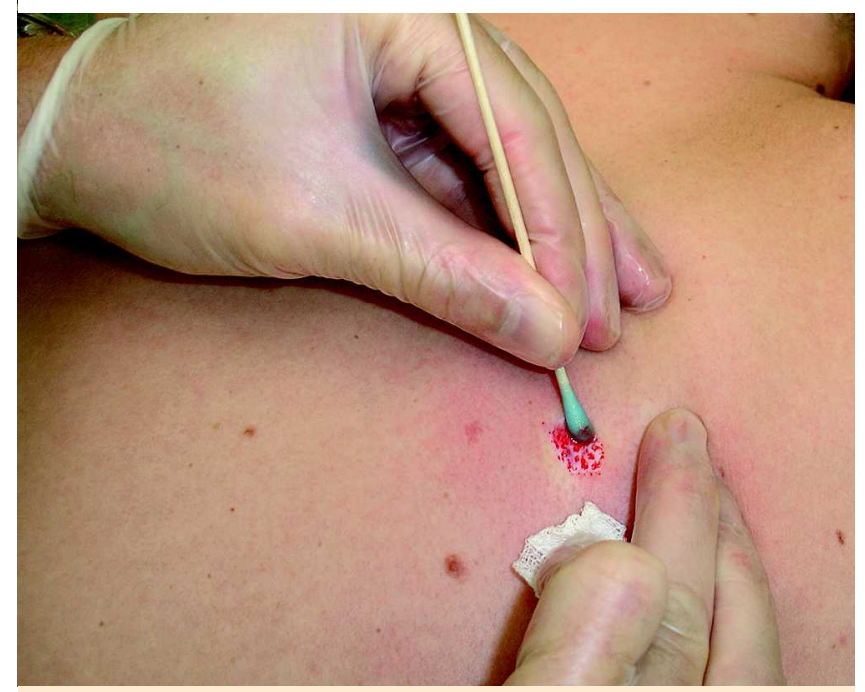

Abb. 3 Blutstillung mit Mallebrin ${ }^{\circledR}$-getränktem Stieltupfer.

\section{Blutstillung}

Nach der Shave-Exzision treten Punktblutungen auf. Die Blutstillung erfolgt mit einem kleinen Stieltupfer, der in eine Flasche mit Aluminiumchlorid-Konzentrat (Mallebrin ${ }^{\circledR}$, normalerweise zum Gurgeln bei Mundschleimhaut-Entzündungen) getunkt und anschließend über die Wunde gerieben wird ( $\triangle$ Abb. $\mathbf{3}$ ). Das durch Mallebrin ${ }^{\circledR}$ üblicherweise auf Wunden induzierte Brennen entfällt aufgrund der Lokalanästhesie. Mallebrin ${ }^{\circledR}$ funktioniert so gut, dass Shave-Exzisionen auch unter ASS und Marcumar $^{\circledR}$ durchgeführt werden können. Wiedervorstellungen und umständliche Marcumar ${ }^{\circledR}$-Pausen mit Heparinisierung entfallen hierdurch.

\section{Nachbehandlung der Shave-Wunde} $\nabla$

Zur Wundbehandlung eignet sich Fusidinsäure-Salbe (Fucidine $^{\circledR}$ ). Aufgeklärt wird über die sekundäre Wundheilung, dass nach initialer Rötung nachfolgend ein gelblicher Belag entsteht, ein Fibrin-Belag, kein Eiter. Analgetika sind bei Shave-Exzisionen postoperativ i.d.R. verzichtbar. Nachkontrollen in der Praxis sind ebenfalls entbehrlich. Duschen ist am Folgetag bereits erlaubt, ebenso jede Form von Sport. Die Histologie kann vom Patienten ggf. 10 Tage postoperativ telefonisch erfragt werden. 


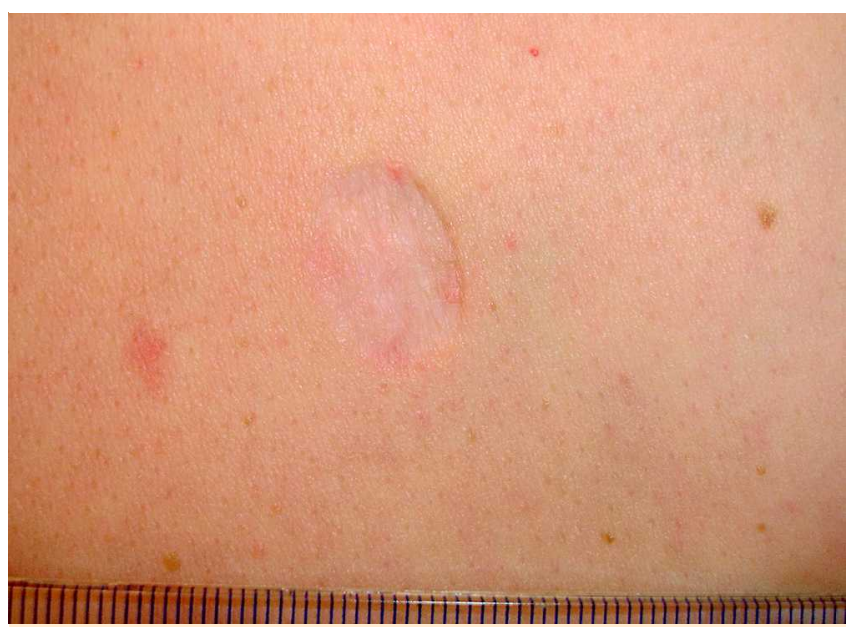

Abb. 4 Klinischer Befund sechs Monate nach Durchführung der ShaveExzision.

Erfolgt die Wiedervorstellung wegen protrahierter Abheilung, kann die Wundbehandlung ausnahmsweise mit einer Hydrokolloid-Platte wie beispielsweise Algoplaque Film ${ }^{\circledR}$ erfolgen. Nach einer Shave-Exzision pektoral kann ein Kontroll-Termin 6 Wochen später sinnvoll sein, denn die Komplikation eines Keloids wäre am ehesten hier zu erwarten.

\section{Diskussion}

$\nabla$

Dysplastische NZN können häufig wie beschrieben durch ShaveExzisionen entfernt werden: Mit wenig Vorbereitung, geringem Zeitaufwand und in der Regel gutem ästhetischen Ergebnis. Die Reepithelialisierung erfolgt vom Wundrand und von den Haarfollikeln, und die Rate narbenloser Abheilung wird mit 9,7\% von 403 melanozytären Nävi [2] bzw. 37\% von 82 papillomatösen Nävi angegeben [5]. Komplikationen wie Wundinfektionen oder Keloide werden mit 7,9\% von 403 durchgeführten ShaveExzision melanozytärer Nävi seltener angegeben als bei $15 \%$ von 268 konventionellen Spindelexzisionen [2]. Neben Hypertrophie oder Keloiden ist gelegentlich mit Hypo- oder Hyperpigmentierung oder einer leichten Mulde als Ergebnis zu rechnen ( $\bullet$ Abb. 4). Häufiger resultieren aber immer noch bessere ästhetische Ergebnisse als z.B. dehiszente längere Narben am Rücken als Folge nach konventionellen Spindelexzisionen. Dies erklärt die hohe subjektive Zufriedenheit nach Shave-Exzisionen [1,2]. Kontrovers diskutiert wird bei Shave-Exzisionen die oft knappe Entfernung mit der Gefahr des Nävusrezidivs: Es wird mit insgesamt 18,1\% [2] bzw. 28\% [1] angegeben, bei Compoundnävi und intradermalen Nävi häufiger als bei Junktionsnävi, im Vergleich zu 6\% bei konventionellen Spindelexzisionen [2]. Rezidive nach Shave-Exzisionen treten meistens im Zentrum der Shave-Exzisionen auf. Durch Schnittführung in der richtigen Ebene nach unmittelbar zuvor konsequentem Aufpolstern der Dermis mit Lokalanästhetikum wie in diesem Beitrag beschrieben lässt sich die Rate dieser Komplikation gering halten. Dem dermatohistologischen Befund ist zu entnehmen, ob die Läsion vollständig oder nur randbildend entfernt wurde. Zeigen sich histologisch Dysplasie und Randbildung, ist der Patient zur klinischen Kontrolle der Shave-Stelle in 2-3 Monaten einzubestellen und bei ausbleibender Repigmentierung auf die Rezidiv-Gefahr und die dann notwendige Wiedervorstellung aufmerksam zu machen.
Ergibt die Histologie schwere Dysplasie oder wider Erwarten sogar ein (In-situ-)Melanom, ist eine konventionelle Nachexzision indiziert. Bei klinisch melanomverdächtigen Befunden verbieten sich Shave-Exzisionen. Ein „worst case“, das Entstehen eines Melanoms infolge randbildender Shave-Exzision eines dyplastischen NZN, ist bisher nicht publiziert, trotz weiter Verbreitung der Technik insbesondere im englischen Sprachraum [3 -7].

Auch für Sondersituationen mögen Shave-Exzisionen infrage kommen. So kann z. B. bei Kleinkindern, die bei einer konventionellen Exzision z.B. eines Spitz-Nävus oder bei einer Probeexzision eines klinisch unklaren Befundes nicht ausreichend kooperieren würden, eine zügig durchgeführte Shave-Exzision möglich sein. Eine Vollnarkose wegen des Alters kann hierdurch vermieden werden.

Im Alltag vieler Kollegen bewährt sich jedenfalls diese Operations-Technik, die dem zunehmenden Wunsch der Patienten nach minimal-invasiven Eingriffen entgegenkommt und auch in Zeiten von hoher Arbeitsbelastung, Niedrighonoraren und Budgetierung effektiv und wirtschaftlich erbracht werden kann, mit zumeist gutem ästhetischen Ergebnis. Nicht weniger als bei konventionellen Spindelexzisionen erfordert dieses weniger traumatisierende Verfahren Erfahrung und Geschick.

\section{Abstract}

\section{Shave Excisions of Dysplastic Nevi: Methodical Approach $\nabla$}

Excision of dysplastic nevus as the most frequent surgery of the dermatologist can be conducted minimally invasive as shave excision. In the present report, technical aspects are reviewed: Such as the bolstering up of the dermis by local anaesthetics to hit the right excision level, not too flat because of risk of relapse, not too deep because of risk of unaesthetic scar. A rapid haemostasis is achieved by aluminium chloride solution (Mallebrin ${ }^{\circledR}$ ), the wound subsequently may be treated with fusidinic acid (Fucidine ${ }^{\circledR}$ ). Pause with coumarine and heparin treatment, general anaesthesia for infants or ban on sports following surgery are not necessary when shave excisions are done. When dysplasia is described in histology, place of surgery will be checked two or three months later and patient will be informed of the risk of nevus recurrence. The less traumatizing procedure of shave excision can be carried out easily immediately following preventive skin cancer check-up and in the hands of a skilled dermatologist mostly achieves good aesthetic result.

\section{Literatur}

1 Bong JL, Perkins $W$. Shave excision of benign facial melanocytic naevi, a patient's satisfaction survey. Dermatol Surg 2003; 29: 227-229

2 Breuninger H, Garbe C, Rassner G. Shave-Exzision von melanozytären Nävi der Haut. Hautarzt 2000; 51: 75-580

3 Harrison PV. Good results after shave excision of benign moles. J Dermatol Surg Oncol 1985; 11: 668

4 Hudson-Peacock MJ, Bishop J, Lawrence CM. Shave excision of benign papular naevocytic naevi. Br J Plast Surg 1995; 48: 318 - 322

5 Hudson-Peacock MJ, Lawrence CM. Outcome of shave excision of benign compound nevi. Br J Dermatol 1994; 131 (Suppl 44): 23

6 Piepkorn MW, Meyer LJ, Goldgard D. The dysplastic melanocytic nevus, a prevalent lesion that correlates poorly with clinical phenotype. J Am Acad Dermatol 1989; 20: 407-415

7 Porter JM, Treasure J. Excision of benign pigmented skin tumours by deep shaving. Br J Plast Surg 1993; 46: 255-257 